\title{
Endovascular Dural Venous Sinus Stenting for the Treatment of Papilledema in Idiopathic Intracranial Hypertension
}

\author{
Taylor G Maloney ${ }^{1 *}$, Joseph Oros ${ }^{2}$, Reshma George ${ }^{2}$, Ryan Surujdin ${ }^{1,2}$, Connor Gilliland ${ }^{3}$ and \\ Joseph H Hise ${ }^{1}$
}

${ }^{1}$ Depatment of Radiology, Baylor University Medical Center, Dallas, TX

${ }^{2}$ Texas A\&M Health Science Center College of Medicine, Dallas, TX

${ }^{3}$ Baylor University, Waco, $T X$

\begin{abstract}
Introduction: Recently a subset of patients with Benign Intracranial hypertension has been identified that have cerebral dural venous stenosis. Recent studies have demonstrated that venous sinus stenting can result in normalization of these pressures and resultant resolution of ophthalmologic findings.

Methods: Twenty-six patients were referred to a single institution for optic nerve swelling due to Benign Intracranial hypertension and found to have cerebral dural venous sinus stenosis. These patients subsequently underwent cerebral venous sinus stenting. Ophthalmic manifestations were evaluated pre and post procedure as well as intravascular pressure measurements and pressure gradients before and after the stenting procedure. The pre and postoperative ophthalmologic findings were tabulated as well as the pre and post procedure pressure measurements before and after stenting.
\end{abstract}

Results: The average preprocedural pressure gradient measured $13.8 \mathrm{mmHg}$ and was reduced to $1.3 \mathrm{mmHg}$ after the stenting procedure. 25 of 26 patients had improvement in their papilledema after the stenting procedure. No complications occurred during or after the procedure.

Conclusion: Endovascular Dural Venous sinus stenting is a safe and effective treatment modality for the ophthalmic manifestations of Benign Intracranial Hypertension in those patients found to have cerebral venous sinus stenosis.

\section{Introduction}

Idiopathic intracranial hypertension (IIH) is well known but poorly understood condition that can have devastating consequences on patients. It is a disorder in which the intracranial pressure (ICP) is abnormally increased in the absence of an underlying mass lesion. IIH, formerly known as pseudotumor cerebri, classically affects women of childbearing age with increased BMI. Symptoms of IIH include headaches, tinnitus, and visual problems. Papilledema can develop in severe cases and is associated with progressive vision loss [1]. The visual loss can even occur despite medical and surgical intervention. The elevated intracranial pressures can cause pressure on and swelling of the prelaminar optic nerve resulting in papilledema and subsequent visual loss1 [1]. Ophthalmologic studies have demonstrated the association of optic nerve swelling with elevated intracranial pressures [2]. Ophthalmic evaluation of the patient with Idiopathic Intracranial hypertension includes visual acuity assessment, funduscopic evaluation and frequently retinal photography, automated perimetry for peripheral visual field assessment, and occasionally Optical Coherence Tomography (OCT). OCT is a newer methodology that delineates the thickness of the retinal nerve fiber layer (RNFL) which may be increased in acute cases of IIH or demonstrate thinning with chronic cases of IIH $[3,4]$. Thinning of the RNFL has been correlated with visual changes on automated perimetry testing [5]. These findings frequently occur in the setting of a patient with retrobulbar pain with or without subjective visual changes.

*Corresponding author: Taylor Maloney, Department of Radiology, 3500 Gaston Avenue Dallas, TX 75246, Tel: 214-8200111, USA

Accepted: June 11, 2021

Published online: June 14, 2021

Citation: Maloney TG, Oros J, George R, et al. (2021) Endovascular Dural Venous Sinus Stenting for the Treatment of Papilledema in Idiopathic Intracranial Hypertension. J Ophthalmic Surg 4(1):27-29 
Citation: Maloney TG, Oros J, George R, et al. (2021) Endovascular Dural Venous Sinus Stenting for the Treatment of Papilledema in Idiopathic Intracranial Hypertension. J Ophthalmic Surg 4(1):27-29

Symptomatically, Benign Intracranial Hypertension can cause debilitating headaches. These headaches may or may not be responsive to medical therapy. Medical therapy with Carbonic Hydrase inhibitors such as Acetazolamide has variable success in relieving the elevated intracranial pressure, the papilledema and the subjective complaints of visual disturbances and even headache. Other well recognized treatment modalities include cerebral shunting procedures (VP and LP shunt) and Optic nerve sheath fenestration (ONSF) [6].

The pathogenesis of $\mathrm{IIH}$ is poorly understood and likely multifactorial, but it is known that hypertrophied arachnoid granulations can exert mass effect on the Dural venous sinus lumen and cause a focal stenosis. $2[7,8]$. These focal stenoses can cause venous outflow obstruction which leads to increased intracranial venous pressure, increased ICP, and ultimately papilledema [8-10]. The goal of endovascular dural venous sinus stenting (DVSS) in select IIH patients is to eradicate these focal stenoses, correct their intracranial pressure, and resolve their symptoms. Endovascular DVSS is becoming a more commonly accepted treatment for IIH [811]. We describe our institutional experience and findings with associated with endovascular dural venous sinus stenting.

\section{Materials}

Institutional IRB approval was obtained prior to the study from Baylor University Medical Center's IRB panel. Patients were selected based on referrals from Ophthalmologists that identified IIH patients with papilledema and possible cerebral dural venous sinus stenosis. All patients were evaluated neuroradiologically at Baylor University Medical Center. 26 patients ( 25 females, 1 male) with evidence of papilledema secondary to idiopathic intracranial hypertension presented to a single center between 3/2016-5/2019 for dural venous sinus stenting. These patients had an average age of 35.4 (range 18-53) with papilledema on initial fundoscopic examination. MR or CT venogram was then performed demonstrating dural venous sinus stenosis. The patients were then referred to the radiology department for catheter venogram and dural venous sinus stenting. Venous pressure measurements were taken proximal to and distal to the stenosed section before and after stent deployment. All procedures were performed at a single center in a biplane neuro-interventional suite. Initial and follow-up ophthalmic evaluations and fundoscopic examinations were performed by the referring clinician.

\section{Results}

Mean pre-treatment pressure gradient across the stenosed section was $13.8 \mathrm{mmHg}$ (range $7-28 \mathrm{mmHg}$ ). Mean post-treatment pressure gradient section was 1.3 $\mathrm{mmHg}$ (range 0-4), with a reduction in the mean pressure gradient by $12.0 \mathrm{mmHg}$. No major or minor complications were reported during or after the procedures. Follow up fundoscopic examination demonstrated interval resolution of papilledema in 25 of 26 patients. Two patients had late recurrence (months) of their papilledema; repeat venography on these patients demonstrated stenoses distal to the existing stent and were re-treated with angioplasty and stenting with ultimate resolution of their optic nerve swelling.
The average BMI of our patient population was 36.5 (range 20.2 to 62.1). If the patient had a history of prior Dural venous sinus thrombosis, web formation often occurred which necessitates angioplasty prior to stent deployment. The authors note that if angioplasty is not performed in those patients with history of dural venous sinus thrombosis, the deployed stent will often have a narrow waist which prevents complete stent expansion without angioplasty.

\section{Discussion}

Idiopathic intracranial hypertension most likely is a symptom manifestation of a variety of conditions that result in elevated intracranial pressure resulting in the typical symptomatology associated with the condition. This study highlights the possibility that the underlying etiology may indeed be related to an anatomic condition - narrowing of the cerebral venous sinus'. This may exacerbate the fluid dynamics in the brain resulting in ever increasing intracranial pressures and worsening stenosis [12]. Therefore, MR or CT venogram should be considered in all patients that present with a constellation of findings of optic nerve swelling, elevated intracranial pressures and stigmata of IIH. In these patients, dural venous sinus stenting is a safe and effective treatment for papilledema in appropriately selected patients with idiopathic intracranial hypertension and Dural venous sinus stenosis. This single-center study demonstrates a decrease in both mean trans-stenosis pressure gradient and papilledema after stent deployment across the stenosed dural venous sinus. Dural venous sinus stenting has a critical role in IIH management which necessitates a multidisciplinary approach. Further research in DVSS along with inclusion of criteria such as headache and visual outcomes is warranted to better treat this potentially debilitating condition.

\section{Acknowledgements}

a. No governmental or non-governmental support was provided for this research paper.

b. No conflicts of interest or financial disclosures exist for any of the authors.

c. No other acknowledgements.

\section{Conflicts of interest}

None.

\section{References}

1. Rigi M, Sumayya J Almarzouqi, Michael L Morgan, et al. (2015) Papilledema: Epidemiology, Etiology, and Clinical Management. Eye Brain 7: 47-57.

2. Hayreh SS (2016) Pathogenesis of Optic Disc Edema in Raised Intracranial Pressure. Prog Retin Eye Res 50: 108-44.

3. Monteiro ML, CL Afonso (2014) Macular Thickness Measurements with Frequency Domain-Oct for Quantification of Axonal Loss in Chronic Papilledema from Pseudotumor Cerebri Syndrome. Eye (Lond) 28: 390-398.

4. Moreno-Ajona D, JA McHugh, J Hoffmann (2020) An Update on Imaging in Idiopathic Intracranial Hypertension. Front Neurol 11: 453. 
5. Zangerl B, Andrew Whatham, Juno Kim, et al. (2017) Reconciling Visual Field Defects and Retinal Nerve Fibre Layer Asymmetric Patterns in Retrograde Degeneration: An Extended Case Series. Clin Exp Optom 100: 214-226.

6. Thurtell MJ, M Wall (2013) Idiopathic Intracranial Hypertension (Pseudotumor Cerebri): Recognition, Treatment, and Ongoing Management. Curr Treat Options Neurol 15: 1-12.

7. Leach JL, Jones BV, Tomsick TA, et al. (1996) Normal Appearance of Arachnoid Granulations on Contrast-Enhanced $\mathrm{Ct}$ and $\mathrm{Mr}$ of the Brain: Differentiation from Dural Sinus Disease. AJNR Am J Neuroradiol 17: 1523-1532.

8. Gurney SP, Sateesh Ramalingam, Alan Thomas, et al. (2020) Exploring the Current Management Idiopathic Intracranial Hypertension, and Understanding the Role of Dural Venous Sinus Stenting. Eye Brain 12: 1-13.
9. Dinkin M, C Oliveira (2019) Men Are from Mars, Idiopathic Intracranial Hypertension Is from Venous: The Role of Venous Sinus Stenosis and Stenting in Idiopathic Intracranial Hypertension. Semin Neurol 39: 692-703.

10. Leishangthem L, Pooja SirDeshpande, Dharti Dua, et al. (2019) Dural Venous Sinus Stenting for Idiopathic Intracranial Hypertension: An Updated Review. J Neuroradiol 46: 148-154.

11. Chatterjee, Ritodhi, et al. (2019) An Evolving Therapy - Dural Venous Sinus Stenting for Idiopathic Intracranial Hypertension. Esn - 2057-0155. The Canadian journal of neurological sciences Le journal canadien des sciences neurologiques 46: 469-471.

12. Boddu S, Marc Dinkin, Maria Suurna, et al. (2016) Resolution of Pulsatile Tinnitus after Venous Sinus Stenting in Patients with Idiopathic Intracranial Hypertension. PLoS One 11: e0164466. 